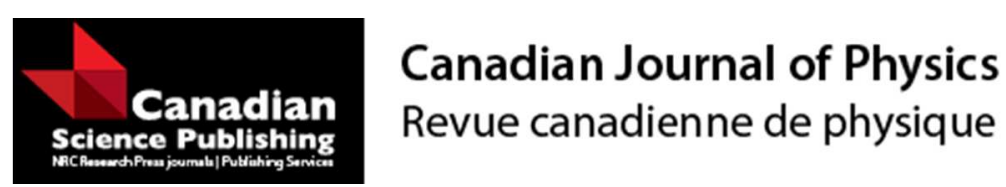

\title{
Collapse and Expansion of Plane Symmetric Charged Anisotropic Source
}

\begin{tabular}{|r|l|}
\hline Journal: & Canadian Journal of Physics \\
\hline Manuscript ID & cjp-2016-0741 \\
\hline Manuscript Type: & Article \\
\hline Date Submitted by the Author: & 11 -Oct-2016 \\
\hline Complete List of Authors: & $\begin{array}{l}\text { Abbas, G.; The Islamia University Bahawalpur } \\
\text { Shah, S.; The Islamia University of Bahawalpur, Mathematics } \\
\text { Zubair, M.; COMSATS Institute of Information Technology, Mathematics }\end{array}$ \\
\hline Keyword: & $\begin{array}{l}\text { Anisotropic Fluids, Electromagnetic Field, Gravitational Collapse, Expanding } \\
\text { solutions, Plane symmetry }\end{array}$ \\
\hline
\end{tabular}

\section{SCHOLARONE ${ }^{\text {m }}$ \\ Manuscripts}




\title{
Collapse and Expansion of Plane Symmetric Charged Anisotropic Source
}

\author{
G. Abbas $^{1}{ }^{*}$ S. M. Shah ${ }^{\dagger}{ }^{\dagger}$ and M. Zubair ${ }^{2} \ddagger$ \\ ${ }^{1}$ Department of Mathematics, The Islamia \\ University of Bahawalpur, Bahawalpur-63100, Pakistan. \\ ${ }^{2}$ Department of Mathematics, COMSATS \\ Institute of Information Technology, Lahore-54000, Pakistan.
}

\begin{abstract}
In this paper, we have investigated the final evolutionary stages of charged non-static plane symmetric anisotropic source. To this end, we have solved the Einstein-Maxwell field equations with the charged plane symmetric source. We have found that vanishing of radial heat flux in the gravitating source provides the parametric form of the metric functions. The new form of the metric functions can generate a class of physically acceptable solutions depending on the choice parameter. These solutions may be classified as expanding or collapsing solutions with the particular values of generating parameter. The gravitational collapse in this case end with the formation of single apparent horizon while there exists two such horizon in case of charged spherical anisotropic source.
\end{abstract}

Keywords: Anisotropic Fluids; Electromagnetic Field; Expansion; Gravitational Collapse.

PACS: $04.20 . \mathrm{Cv} ; 04.20 . \mathrm{Dw}$

*abbasg91@yahoo.com

${ }^{\dagger}$ syedmunawarshah71@hotmail.com

†mzubairkk@gmail.com, drzubair@ciitsahiwal.edu.pk 


\section{Introduction}

In 1939, Oppenheimer and Snyder [1] were the pioneer, who studied the spherically symmetric gravitational collapse of a massive star consisting of dust matter. Initially, it was a particular and simple statement of the gravitational collapse of the star because dust could not be considered as a general realistic matter and the role of matter stress on the formation of singularity cannot be neglected. The general treatment for the problem of gravitational collapse was carried out by Misner and Sharp [2]. They consider the isotropic perfect fluid in the interior region of a star and constructed the equations of motions which governs the perfect fluid gravitational collapse. Further, Misner and Sharp [3] examined the gravitational collapse for anisotropic fluid taking a more realistic form of star geometry. After this there has been an extensive interest among the physicist to discuss the relativistic gravitational collapse of anisotropic gravitating source $[4,5]$.

The analytic solutions of anisotropic sources has achieved much attention in General Relativity due to their implication in astronomy as astrophysics [6]-[10]. Barcelo et al.[11] have investigated the evolution of anisotropic sources in quantum gravity and proposed a new model of gravitational collapse without horizons and trapped surfaces. Many researchers [11]-[15] have investigated the anisotropic behavior of dark energy fluid source in modified $f(R)$ gravity. Further, Herrera and Santos [16] have studied the models of gravitational collapse of massive stars and pointed out the factors affecting stable state of stars under the linear perturbation. Herrera et al. [4] proposed a single generating function in the modeling of anisotropic source, we have used such generating function in the present paper for the modeling of charged anisotropic plane symmetric source.

The generating solution approach for the non-adiabatic gravitating source collapse was proposed for the first time by Glass [17]. For this purpose, he utilized adiabatic fluid static/nonstatic solutions of shearfree collapsing model to a shear-free collapsing source which has dissipation in the form of outward radial flow. Recently, Glass [18] has determined the anisotropic perfect fluid spherically symmetric gravitating model. Abbas and his collaborators [19][22] have generalized this work for the plane symmetric anisotropic source and charged anisotropic spherical and cylindrical sources. In this paper, we examined the effects of electric charge parameter on the dynamics of plane symmetric anisotropic source. The geometries with plane shapes would be interesting models for numerical relativity, quantum gravity, cosmic censor- 
ship hypothesis and hoop conjuncture. Sharif and Zaeem [23] have proposed that plane symmetric gravitating sources are viable as compared to spherical models for determining the different evolutionary stages of our universe. The present paper is a systematic study of Glass [18] work to discuss the evolution of the charged plane symmetric source.

This paper has been arranged as follows: In the next section, we drive the equations of motions of charged anisotropic plane symmetric source. Section 3 deals with the parametric solutions of the field equations which exhibit the evolution of the source as expansion or collapse depending on the choice of parameters of the proposed model. The last section contains the summary of results.

\section{Gravitating Source and Einstein-Maxwell Field Equations}

The plane symmetric anisotropic charged fluid source is defined by the following line element

$$
d s_{-}^{2}=-X^{2}(t, z) d t^{2}+Y^{2}(t, z)\left(d x^{2}+d y^{2}\right)+Z^{2}(t, z) d z^{2} .
$$

The charged anisotropic source has following form of stress energy tensor

$$
\begin{aligned}
T_{a b} & =\left(\mu+P_{\perp}\right) U_{a} U_{b}+P_{\perp} g_{a b}+\left(P_{z}-P_{\perp}\right) \psi_{a} \psi_{b}+p_{r} g_{\alpha \beta} \\
& +\frac{1}{4 \pi}\left(F_{a}^{c} F_{b c}-\frac{1}{4} F^{c d} F_{c d} g_{a b}\right)
\end{aligned}
$$

here $P_{z}$ and $P_{\perp}$ are pressures along $z$-direction and perpendicular direction, $\psi^{a}$ is a four vector along $z$-direction and $U_{a}$ is a co-moving four velocity. Further, $F_{a b}=-\phi_{a, b}+\phi_{b, a}$ is electromagnetic field tensor with four vector potential $\phi_{a}$.

The Maxwell field equations are

$$
F_{; \beta}^{\alpha \beta}=4 \pi J^{\alpha}, \quad F_{[\alpha \beta ; \gamma]}=0,
$$

here, we can define the four-potential and four-current as follows:

$$
\phi_{\alpha}=\phi \delta_{\alpha}^{0}, \quad J^{\alpha}=\varrho U^{\alpha},
$$


where $\varrho$ and $\phi$ are electric charge density and electric scalar potential, respectively.

The expansion scalar is

$$
\Theta=\frac{1}{X}\left(\frac{2 \dot{Y}}{Y}+\frac{\dot{Z}}{Z}\right)
$$

Here dot is partial derivative with to time coordinate $t$. The fractional anisotropy of the fluid is defined as

$$
\Delta a=1-\frac{P_{\perp}}{P_{z}}
$$

The set of Einstein field equations is given by

$$
\begin{aligned}
8 \pi \mu X^{2}+\frac{16 \pi^{2} Q^{2} X^{2}}{Y^{4}} & =\frac{\dot{Y}}{Y}\left(\frac{2 \dot{Z}}{Z}+\frac{\dot{Y}}{Y}\right)+\left(\frac{X}{Z}\right)^{2}\left(\frac{-2 Y^{\prime \prime}}{Y}+\left(\frac{2 Z^{\prime}}{Z}-\frac{X^{\prime}}{X}\right) \frac{X^{\prime}}{X}\right), \\
& -\frac{\dot{Y}^{\prime}}{Y}+\frac{X^{\prime} \dot{Y}}{X Y}+\frac{\dot{Z} Y^{\prime}}{Y Z}=0, \\
8 \pi P_{\perp} Y^{2}+\frac{16 \pi^{2} Q^{2}}{Y^{2}} & =-\left(\frac{Y}{X}\right)^{2}\left[\frac{\ddot{Y}}{Y}+\frac{\ddot{Z}}{Z}-\frac{\dot{X}}{X}\left(\frac{\dot{Y}}{Y}+\frac{\dot{Z}}{Z}\right)+\frac{\dot{Y} \dot{Z}}{Y Z}\right] \\
& +\frac{Y^{2}}{Z^{2}}\left[\frac{X^{\prime \prime}}{X}+\frac{Y^{\prime \prime}}{Y}-\frac{X^{\prime}}{X}\left(\frac{Z^{\prime}}{Z}-\frac{Y^{\prime}}{Y}\right)-\frac{Y^{\prime} Z^{\prime}}{Y Z}\right], \\
8 \pi P_{z} Z^{2}-\frac{16 \pi^{2} Q^{2} Z^{2}}{Y^{4}} & =-\left(\frac{Z}{X}\right)^{2}\left[\frac{2 \ddot{Y}}{Y}+\left(\frac{\dot{Y}}{Y}\right)^{2}-\frac{2 \dot{X} \dot{Y}}{X Y}\right] \\
& +\left(\frac{Y^{\prime}}{Y}\right)^{2}+\frac{2 X^{\prime} Y^{\prime}}{X Y} .
\end{aligned}
$$

By the direct calculation, we note that if $X$ and $Z$ have following functional form

$$
X=\frac{Y}{Y^{\alpha}} \quad Z=Y^{\alpha}
$$

then Eq.(4), can be satisfied easily. By Eq.(4), expansion scalar takes the following form

$$
\Theta=(2+\alpha) Y^{(1-\alpha)} \text {. }
$$


It is interesting to mention here that if $\alpha>-2$ and $\alpha<-2$, then the evolution of source may results as expansion and collapse.

Hence, by Eq.(10), we get the following form of Einstein field equations

$$
\begin{aligned}
8 \pi \mu+\frac{Q^{2} \dot{Y}^{2}}{Y^{2 \alpha+4}} & =(1+2 \alpha) Y^{2 \alpha-2}-\frac{1}{Y^{2 \alpha}}\left[\frac{2 Y^{\prime \prime}}{Y}+(1-2 \alpha)\left(\frac{Y^{\prime}}{Y}\right)^{2}\right]+\frac{1}{Y^{2}}, \\
8 \pi P_{z}-\frac{16 \pi^{2} Q^{2} Y^{2 \alpha}}{Y^{4}} & =(1+2 \alpha) Y^{2 \alpha-2}+\frac{1}{Y^{2}}+\frac{1}{Y^{2 \alpha}}\left[(2 \alpha-1)\left(\frac{Y^{\prime}}{Y}\right)^{2}-\frac{2 \dot{Y}^{\prime}}{\dot{Y}} \frac{Y^{\prime}}{Y}\right], \\
8 \pi P_{\perp}+\frac{16 \pi^{2} Q^{2}}{Y^{4}} & =-\alpha(1+2 \alpha) Y^{2 \alpha-2} \\
& +\frac{1}{Y^{2 \alpha}}\left[(1-\alpha) \frac{Y^{\prime \prime}}{Y}-(3 \alpha-1) \frac{\dot{Y}^{\prime}}{\dot{Y}}\left(\frac{Y^{\prime}}{Y}\right)+\frac{\dot{Y}^{\prime \prime}}{Y}+\alpha(2 \alpha-1)\left(\frac{Y^{\prime}}{Y}\right)^{2}\right] .
\end{aligned}
$$

The mass function defined by Taub [24] for plane symmetry with the contribution of electromagnetic field is

$$
m(z, t)=\frac{\left(g_{11}\right)^{3 / 2}}{2} R_{12}^{12}+\frac{Q^{2}}{2 Y} .
$$

Plugging the values of $g_{11}$ and $R_{12}^{12}$ from given spacetime along with $X=$ $\frac{Y}{Y^{\alpha}}, \quad Z=Y^{\alpha}$, the Taub's mass takes the following form

$$
\frac{2 m(t, z)}{Y}-\frac{Q^{2}}{Y^{2}}=\left(Y^{2 \alpha}-\frac{Y^{\prime 2}}{Y^{2 \alpha}}\right)
$$

For $Y^{\prime}=Y^{2 \alpha}$, one gets $Y=\frac{Q^{2}}{2 m}$, which implies that in this case a trapping surface corresponding to single horizon exist at $Y=\frac{Q^{2}}{2 m}$. The trapping condition $Y^{\prime}=Y^{2 \alpha}$, has the integral

$$
Y_{\text {trap }}^{(1-2 \alpha)}=z(1-2 \alpha)+g(t)
$$

where $g(t)$ is the function of integration.

\section{Parametric Solutions}

In this section, we determine the values of parameter $\alpha$, for solutions reveal the expansion and collapse of the source. 


\subsection{Gravitational Collapse for $\alpha=-\frac{5}{2}$}

We know that for a collapsing object, the expansion scalar should be negative. Therefore, we investigate that $\Theta<0$ ( see Eq.(4)), if $\alpha<-2$.Here, without the loss of generality, we take $\alpha=-\frac{5}{2}$ and trapping condition $Y^{\prime}=Y^{2 \alpha}$, becomes $Y^{\prime}=Y^{-5}$, which further leads to

$$
Y_{\text {trap }}=\left(6 z+g_{1}(t)\right)^{\frac{1}{6}}
$$

where $g_{1}(t)$ is function of integration. For $\alpha=-\frac{5}{2}$, Eqs.(6), (7) and (8) give

$$
\begin{aligned}
8 \pi \mu+16 \pi^{2} Q^{2} \dot{Y}^{2} Y & =-4 Y^{-7}-2 Y^{5}\left[\frac{2 Y^{\prime \prime}}{Y}+3\left(\frac{Y^{\prime}}{Y}\right)^{2}\right]+\frac{1}{Y^{2}}, \\
8 \pi P_{z}-\frac{16 \pi^{2} Q^{2}}{Y^{9}} & =-4 Y^{-7}+\frac{1}{Y^{2}}-2 Y^{5}\left[3\left(\frac{Y^{\prime}}{Y}\right)^{2}-\frac{2 \dot{Y}^{\prime}}{\dot{Y}} \frac{Y^{\prime}}{Y}\right], \\
8 \pi P_{\perp}+\frac{16 \pi^{2} Q^{2}}{Y^{4}} & =-10 Y^{-7}+Y^{5}\left[\frac{7}{2} \frac{Y^{\prime \prime}}{Y}+\frac{17}{2} \frac{\dot{Y}^{\prime}}{\dot{Y}}\left(\frac{Y^{\prime}}{Y}\right)+\frac{\dot{Y}^{\prime \prime}}{Y}+15\left(\frac{Y^{\prime}}{Y}\right)^{2}\right] .
\end{aligned}
$$

In order to get a class of solutions, we take $Y_{\text {trap }}=k\left(6 z+g_{1}(t)\right)^{\frac{1}{6}}$, the above equations in this case reduces to

$$
\begin{aligned}
8 \pi \mu & =\left(6 z+h_{1}\right)^{-1 / 3}-\frac{8 \pi^{2} Q^{2}}{3} \dot{h}_{1}^{2}\left(6 z+h_{1}\right)^{3 / 2} \\
8 \pi P_{z} & =\left(6 z+h_{1}\right)^{-1 / 3}+Q^{2}\left(6 z+h_{1}\right)^{-3 / 2} \\
8 \pi P_{\perp} & =Q^{2}\left(6 z+h_{1}\right)^{-1 / 3}
\end{aligned}
$$

The dimensionless measure of anisotropy defined by Eq.(5) is

$$
\triangle a=1+\frac{Q^{2}\left(6 z+h_{1}\right)^{5 / 6}}{Q^{2}\left(6 z+h_{1}\right)^{-1 / 3}+\left(6 z+h_{1}\right)^{5 / 6}} .
$$

It is noted that for $\alpha=\frac{-5}{2}$, we get $\Theta<0$ and energy density remains positive and goes on increasing to attains a finite value for the specific value of charge parameter $Q$ and time dependent function $g_{1}(t)$. As $g_{1}=1+t$, so $\dot{g}_{1}=1$, and the electric charge term in case contributes (being the multiple of $\left.\dot{g}_{1}\right)$ to effect the energy density of the fluid. In this way energy density of 

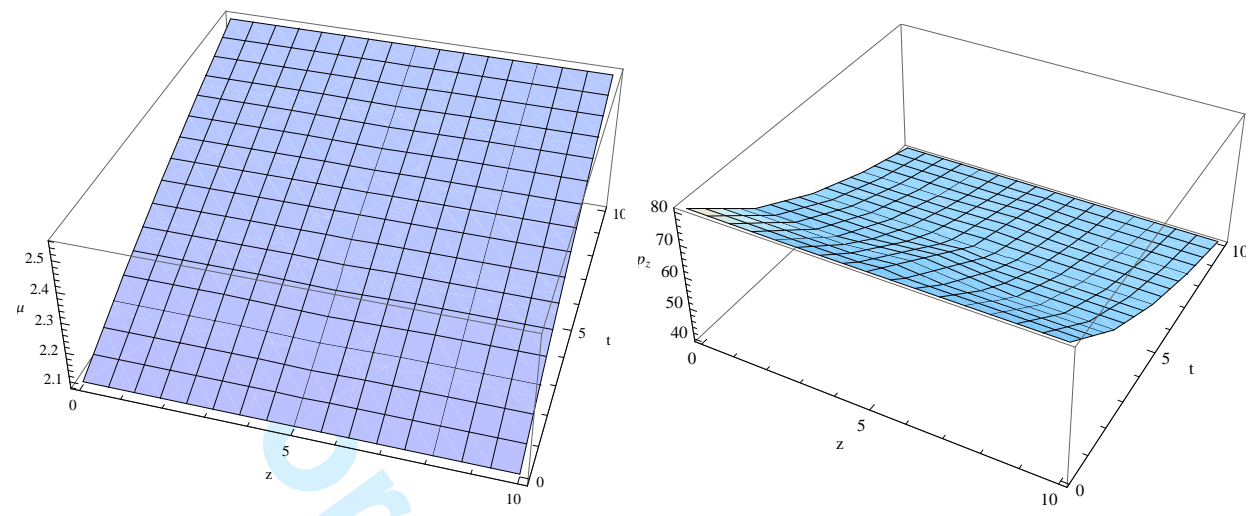

Figure 1: Both graphs have been plotted for $Q=2, g_{1}=1+t$.

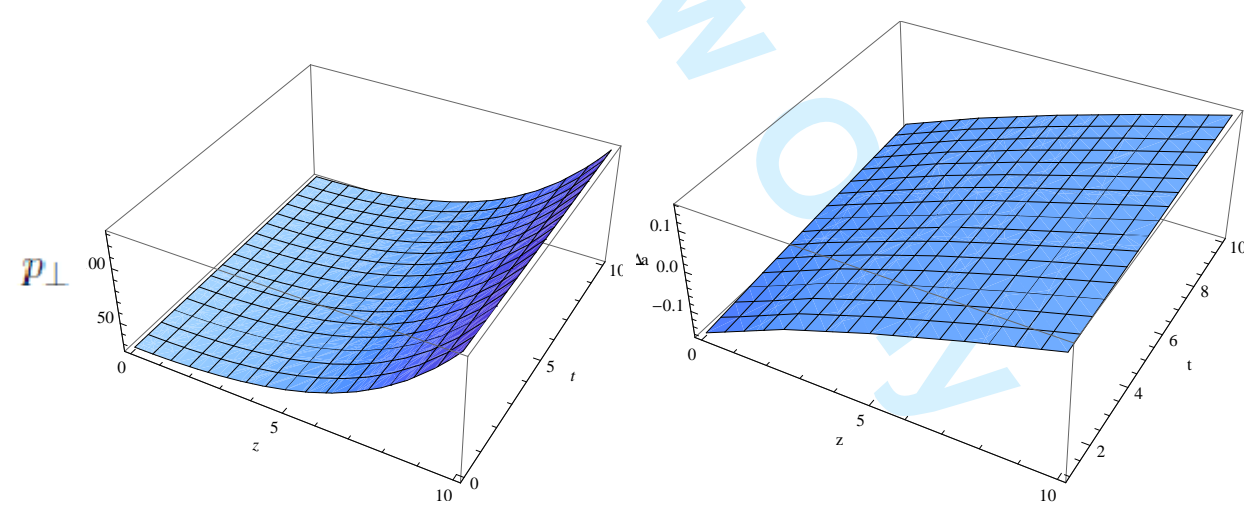

Figure 2: Both graphs have been plotted for $Q=2, g_{1}=1+t$. 
the gravitating source becomes homogeneous and it increases with the time as shown in left graph of Fig.1. Similarly, for the above choice of temporal profile, the pressure $P_{z}$ is decreasing function of time and homogeneous every where inside the gravitating source as shown in right graph of Fig.1. The transverse pressure $P_{\perp}$ and anisotropic parameter $\triangle a$ (with the above choice of $Q$ and $\left.g_{1}(t)\right)$, become inhomogeneous and time independent as shown in Fig.2. The anisotropy increases from negative value to finite positive value.

\subsection{Expansion for $\alpha=\frac{3}{2}$}

Here, we are interested to find such values of $\alpha$ for which expansion scalar becomes positive. It is noted that for $\alpha \geq 0$, we get $\Theta>0$ (from Eq.(4)). In this case, we take $\alpha=\frac{3}{2}$, and also assume that

$$
Y=\left(z^{2}+z_{0}^{2}\right)^{-1}+g_{2}(t)
$$

where $g_{2}(t)$ is an arbitrary function of time and $z_{0}$ an arbitrary constant.

When $\alpha=3 / 2$, the field equations reduce to

$$
\begin{aligned}
8 \pi \mu & =4 Y-2 Y^{-4}\left(Y^{\prime \prime}-\frac{Y^{2}}{Y}\right)+Y^{-2}-Q^{2} \dot{Y}^{2} Y^{-7} \\
8 \pi P_{z} & =4 Y+Y^{-2}+2 Y^{-5}\left(\left(Y^{\prime}\right)^{2}-\frac{\dot{Y}^{\prime}}{\dot{Y}^{\prime}} Y^{\prime}\right)+Q^{2} Y^{-1} \\
8 \pi P_{\perp} & =-6 Y+Y^{-5}\left(-\frac{1}{2} Y^{\prime \prime} Y-\frac{7}{2} \frac{Y^{\prime} \dot{Y}^{\prime}}{\dot{Y}}+\dot{Y}^{\prime \prime} Y+3\left(Y^{\prime}\right)^{2}\right)-Q^{2} Y^{-4}
\end{aligned}
$$

With $F(t, z)=1+g_{2}(t)\left(z^{2}+z_{0}^{2}\right)$ and $Y=\frac{F}{\left(z^{2}+z_{0}^{2}\right)}$, the density and pressures 


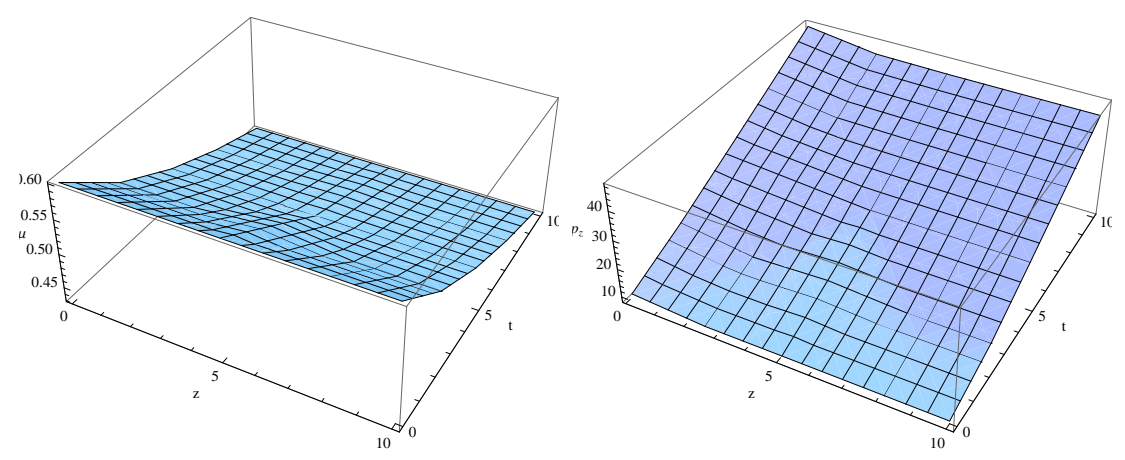

Figure 3: Both graphs have been plotted for $Q=2, g_{2}=1+t$.

can be written as

$$
\begin{aligned}
8 \pi \mu & =\frac{4 F}{\left(z^{2}+z_{0}^{2}\right)}+\frac{\left(z^{2}+z_{0}^{2}\right)^{2}}{F^{2}}+\frac{\left(z^{2}+z_{0}^{2}\right)\left(z_{0}^{2}-3 z^{2}\right)}{F^{4}}+\frac{8 z^{2}\left(z^{2}+z_{0}^{2}\right)}{F^{5}} \\
& -\frac{Q^{2} \dot{h_{1}\left(z^{2}+z_{0}^{2}\right)^{7}}}{F^{7}} \\
8 \pi P_{z} & =\frac{4 F}{\left(z^{2}+z_{0}^{2}\right)}+\frac{\left(z^{2}+z_{0}^{2}\right)^{2}}{F^{2}}+\frac{8 z^{2}\left(z^{2}+z_{0}^{2}\right)}{F^{5}}+\frac{Q^{2}\left(z^{2}+z_{0}^{2}\right)}{F} \\
8 \pi P_{\perp} & =\frac{-6 F}{\left(z^{2}+z_{0}^{2}\right)}+\frac{\left(z^{2}+z_{0}^{2}\right)\left(z_{0}^{2}-3 z^{2}\right)}{F^{4}}+\frac{12 z^{2}\left(z^{2}+z_{0}^{2}\right)}{F^{5}} \\
& -\frac{Q^{2}\left(z^{2}+z_{0}^{2}\right)^{4}}{F^{4}} .
\end{aligned}
$$

The anisotropy (Eq.(5)) can be written as

$\triangle a=1+\frac{\frac{6 F^{6}}{\left(z^{2}+z_{0}^{2}\right)^{2}}-12 z^{2}-F^{3}\left(z^{2}+z_{0}^{2}\right)+F\left[z^{6} Q^{2}-z_{0}^{2}+3 z^{4} Q^{2} z_{0}^{2}+3 z^{2}\left(1+Q^{2} z_{0}^{2}\right)\right]}{\left(\frac{4 F^{6}}{\left(z^{2}+z_{0}^{2}\right)^{2}}+F^{4} Q^{2}+8 z_{0}^{2}+F^{3}\left(z^{2}+z_{0}^{2}\right)\right)}$.

All these quantities are shown graphically in figures $\mathbf{3}$ and $\mathbf{4}$.

It is interesting for $\alpha=\frac{3}{2}$, we get $\Theta>0$ and energy density remains positive and decreases to a finite value for the specific value of charge parameter $Q$ and time dependent function $g_{2}(t)$. As $g_{2}=1+t$, so $\dot{g}_{2}=1$, and the electric charge term in case contributes (being the multiple of $\dot{g}_{1}$ ) to effect the energy density of the fluid. In this way energy density of the gravitating 


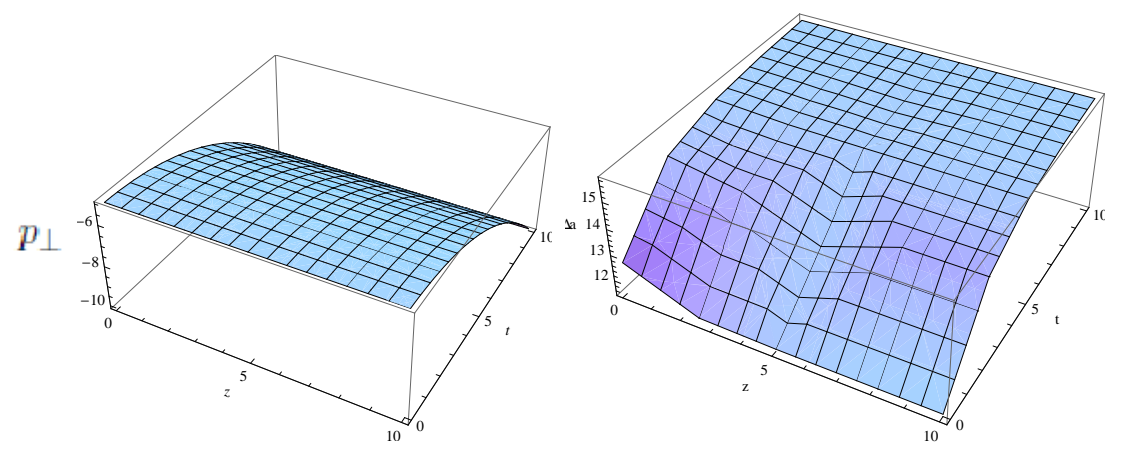

Figure 4: Both graphs have been plotted for $Q=2, g_{2}=1+t$.

source becomes homogeneous and it decreases with the time as shown in left graph of Fig.3. The decrease in energy density confirms the expansion of gravitating source. Similarly, for the above choice of temporal profile, the pressure $P_{z}$ is increasing function of time and homogeneous as shown in right graph of Fig.3. The transverse pressure $P_{\perp}$ and anisotropic parameter $\triangle a$, become homogeneous and time dependent as shown in Fig.2. The anisotropy increases as the gravitating source goes on expanding.

\section{Conclusion}

This paper aims to discuss the evolutionary stages of the charged non-static plane symmetric sources. The source fluid is non-radiating with plane symmetry and have electrical conduction property. The off diagonal component of the field equations provides the metric functions in terms of parametric form of another metric function. The Taub's mass in the presence of electric charge has been calculated which helps to determine the trapped surfaces. The trapping condition $Y^{\prime}=Y^{2 \alpha}$, leads to the existence of single horizon at $Y=\frac{Q^{2}}{2 m}$. Thus the non-vanishing charges in the plane gravitating source plays an important rule for the formation of horizon. It is interesting to note that no such horizon exist in non-charged plane gravitating source dynamics.

During the trapping situation, the relation $Y^{\prime}=Y^{2 \alpha}$ must holds and with this relation, we get $\Theta=(\alpha+2) Y^{(1-\alpha)}$. The behavior of $\Theta$ depends on values of $\alpha$, it can be summarized as follows:

- For $\alpha=-2$, we get $\Theta=0$, which corresponds to bouncing of the gravitating source 
- For $\alpha<-2$, we get $\Theta<0$, which corresponds to the collapse of the gravitating source

- For $\alpha \geq 0$, we get $\Theta>0$, which corresponds to expansion of the gravitating source.

We would like to mention here that in case of gravitational collapse, for the particular choice of the electric charge parameter $Q$ and time profile $g_{1}(t)$, the energy density and $P_{z}$ pressure along $z$-direction are increasing and decreasing function of time respectively. Both these quantities also, become homogenous. The $P_{\perp}$ transverse pressure and $\triangle a$ anisotropic parameter are increasing function of $z$, so both are non-homogenous and time independent. Since in this case $P_{z}$ decreases and $P_{\perp}$ increases, therefore, we get nonzero value of the anisotropic parameter $\triangle a$. On the other hand in case of expansion, for the particular choice of the electric charge parameter $Q$ and time profile $g_{2}(t)$, the energy density and pressure imply the reverse behavior as compared to gravitational collapse case. In this case, energy density decreases and pressure along $z$-direction increases with time. Here, both $P_{\perp}$ and $\triangle a$, are homogenous and increasing function of time. Further, the anisotropy of the source becomes zero for the particular choice of the parameters.

\section{References}

[1] J.R., Oppenheimer, H. Snyder: Phys. Rev. 56(1939)455

[2] C. WMisner, D. Sharp: Phys. Rev. 136(1964)B571

[3] C. WMisner, D. Sharp: Phys. Lett. 15(1965)279

[4] L. Herrera, J. Ospino, and A. Di Prisco: Phys. Rev. D77(2008a)027502

[5] L. Herrera, N.O. Santos, and A. Wang: Phys. Rev. D78(2008b)084024

[6] R. L Bowers, and E. P.T. Liang: Astrophys. J. 188(1974)657

[7] S.S. Bayin: Phys. Rev. D26(1982)1262

[8] M. Cosenza, L. Herrera, M. Esculpi, and L. Witten: J. Math. Phys. 22(1981)118 
[9] M. Sharif and G. Abbas : Astrophys. Space Sci. 335(2011)515

[10] H. Bondi: MNRAS 259(1992)365

[11] C. Barcelo, S. Liberati, S. Sonego and S. Visser: Phys. Rev. D77(2008)044032

[12] G. Cognola, E. Elizalde, S. Nojiri, S.D. Odintov and S. Zerbini: Phys. Rev. D75(2007)086002

[13] S. Nojiri and S.D. Odintov: Phys. Lett. B631(2005)1

[14] S. Nojiri, S.D. Odintov and O.G. Gorbunova: J. Phys. A39(2006)6627

[15] M. Gasperini and G. Veneziano: Astropart. Phys. 1(1993)317

[16] L. Herrera and N.O. Santos: Phys. Rep. 286(1997)53

[17] E.N. Glass: Phys. Lett. A86(1981)351

[18] E.N. Glass : Gen. Relativ. Gravit. 45(2013)2661

[19] G. Abbas : Astrophys. Space Sci. 350(2014)307

[20] G. Abbas : Astrophys. Space Sci. 352(2014)595

[21] G. Abbas, Z. Ahmad, and H. Shah: Astrophys. Space Sci. 335(2015)138

[22] T. Mahmood, S. M. Shah, and G. Abbas: Astrophys. Space Sci. $\mathbf{3 5 7}(2015) 56$

[23] M. Sharif and M. Zaeem: Mod. Phys. Lett. A27(2012)1250141

[24] T. Zannias: Phys. Rev. D41(1990)3252. 